\title{
Notes sur le mobilier funéraire de Thoutmosis IV
}

$\S 1$ - Des poignards nubiens dans la KV 43 ?

Notes of the grave goods of Thutmose IV - $\$ 1$ - Nubian daggers in KV 43?

\section{Renaud Pietri}

\section{(2) OpenEdition \\ 1 Journals}

Édition électronique

URL : http://journals.openedition.org/cel/366

DOI : $10.4000 / \mathrm{cel} .366$

ISSN : 2262-208X

Éditeur

École du Louvre

\section{Référence électronique}

Renaud Pietri, « Notes sur le mobilier funéraire de Thoutmosis IV », Les Cahiers de l'École du Louvre [En ligne], 9 | 2016, mis en ligne le 15 décembre 2016, consulté le 17 septembre 2019. URL : http:// journals.openedition.org/cel/366 ; DOI : 10.4000/cel.366

Ce document a été généré automatiquement le 17 septembre 2019.

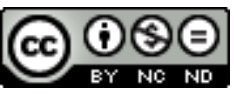

Les Cahiers de l'École du Louvre sont mis à disposition selon les termes de la licence Creative Commons Attribution - Pas d'Utilisation Commerciale - Pas de Modification 4.0 International. 


\title{
Notes sur le mobilier funéraire de Thoutmosis IV
}

\author{
$\S 1$ - Des poignards nubiens dans la KV 43 ? \\ Notes of the grave goods of Thutmose IV - §1 - Nubian daggers in KV 43?
}

\section{Renaud Pietri}

\section{NOTE DE L'AUTEUR}

Cet article est issu de réflexions exposées dans un travail de Master mené à l'École du Louvre en 2011 sous la direction d'Hélène Guichard et de Nathalie Couton-Perche, respectivement conservateur en chef et collaboratrice scientifique au musée du Louvre, cf. Renaud Pietri, Poignards, épées et harpés : de la fabrication au cadeau diplomatique, mémoire de recherche inédit de l'École du Louvre, 2 vol., Paris, 2011, en particulier vol. 1, pp. 65-69. Je souhaite remercier ici Anne-Hélène Perrot et Térésa Ribeyron pour leurs relectures et conseils.

«- Ce n'est pas le stylet de mon père, s'écria

vivement Colomba.

Il a été donné à un des grands-parents de ma mère par le roi Théodore.

Si mademoiselle l'accepte, elle nous fera bien

plaisir.

- Voyez, Miss Lydia, dit Orso, ne dédaignez pas le

stylet d'un roi. »

P. Mérimée, Colomba, 1840.

1 Le mobilier funéraire des rois de la XVIII ${ }^{e}$ dynastie, à l'exception notable de celui de Toutânkhamon dont le degré de représentativité reste toutefois difficile à établir, n'a été que rarement étudié jusqu'à présent et reste mal connu ${ }^{1}$. Peu d'objets ont en effet été retrouvés dans les tombes royales de cette époque, et le passage du temps autant que celui des pillards n'ont souvent laissé aux fouilleurs que de maigres vestiges des 
trésors des rois de cette première moitié du Nouvel Empire, ce qui complique toute étude de synthèse sur le trousseau funéraire royal de la période.

2 Il arrive néanmoins que l'on puisse proposer une hypothèse sur l'un ou l'autre des objets trouvés dans les tombes de la Vallée des Rois : c'est le cas de cette courte note, qui revient sur deux fourreaux trouvés dans la tombe de Thoutmosis IV et s'interroge d'abord sur leur nature puis sur la raison de leur présence au sein du mobilier funéraire de ce prestigieux souverain, fils d'Amenhotep II et père d'Amenhotep III $^{2}$.

Deux fourreaux en cuir ont été découverts dans la tombe de Thoutmosis IV au début du $\mathrm{xx}^{\mathrm{e}}$ siècle, au cours des fouilles menées dans la Vallée des Rois par Howard Carter et Percy E. Newberry. Les deux objets sont aujourd'hui conservés au Musée égyptien du Caire où ils sont inventoriés sous les numéros CG 46114 et CG 46115. Ils ont été publiés en 1904 dans le volume des Catalogue Général du Caire correspondant au mobilier funéraire de la KV $43^{3}$.

4 Le premier objet, qui porte le numéro CG 46114 , mesure environ $13,8 \mathrm{~cm}$ de longueur ${ }^{4}$ pour $5 \mathrm{~cm}$ de largeur maximale et correspond manifestement à la partie supérieure d'un fourreau de poignard (fig.1), comme l'indique la présence de deux languettes d'attache qui permettaient d'accrocher l'étui à une ceinture. La position de ces languettes, qui sont habituellement situées sur les fourreaux juste sous le niveau de la garde du poignard, montre que la protection en cuir devait en fait couvrir tout ou partie de la poignée même de l'arme, un détail significatif sur lequel je reviendrai plus loin. La photographie de l'ouvrage de Carter et Newberry ne montre qu'une seule face $\mathrm{du}$ fourreau, sur laquelle on peut observer une couture centrale.

Fig. 1

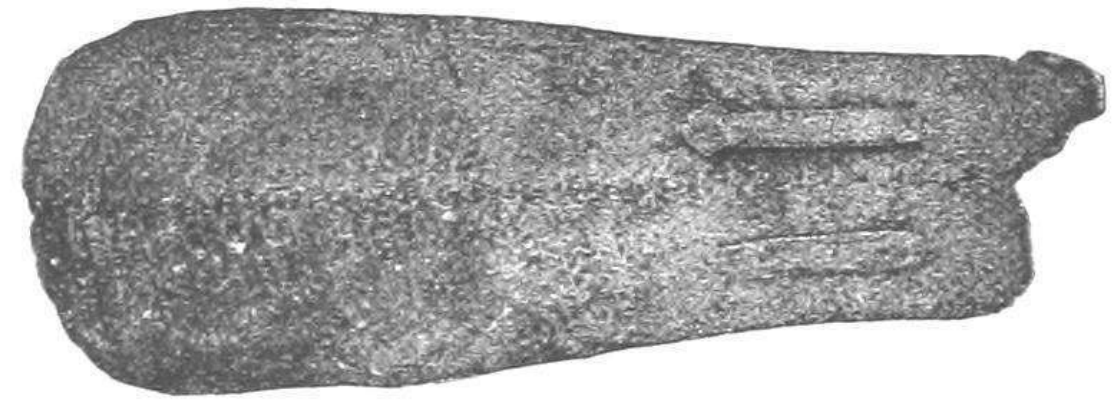

Fragment de fourreau CG 46714 - D'après H. Carter, P. E. Newberry, Catalogue des antiquités égyptiennes du musée du Caire, Nos 46001-46529. The Tomb of Thoutmôsis IV, Westminster, 1904, p. 38, fig. 32.

Le deuxième (fig. 2), numéro CG 46115, semble quant à lui correspondre à un fourreau complet, mais limité cette fois à la lame de l'arme, l'extrémité arrondie et la plus large venant buter contre la garde du poignard. Il mesure $24,5 \mathrm{~cm}$ de long pour une largeur maximale de $3 \mathrm{~cm}$ et comporte également une couture centrale sur toute sa longueur. Un décor constitué de deux lignes parallèles partant de la pointe et se terminant en une ombelle de papyrus qui épouse la bordure supérieure de l'objet vient orner l'étui en léger relief's. Là encore, une seule face de l'objet est montrée dans l'ouvrage de Carter et Newberry. 
Fig. 2

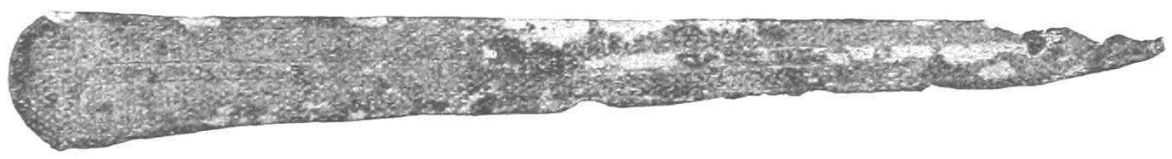

Fragment de fourreau CG 46176 - D’après H. Carter, P. E. Newberry, Catalogue des antiquités égyptiennes du musée du Caire, Nos 46001-46529. The Tomb of Thoutmôsis IV, Westminster, 1904, p. 38, fig. 33.

Le fragment CG 46114 est indiqué comme provenant de la «Room A » de la tombe de Thoutmosis IV, alors que le fourreau CG 46115 aurait été découvert dans la «Chamber 3 ». Ces provenances ne permettent pas d'en dire plus sur l'emplacement d'origine des poignards : la tombe du roi ayant été pillée dans l'Antiquité, les objets du mobilier funéraire ont été dispersés.

7 À ma connaissance, un seul poignard inscrit au nom de Thoutmosis IV nous est parvenu (fig. 3). Conservée au Musée égyptien de Florence ${ }^{6}$, cette arme est réputée provenir de Louqsor où elle aurait été achetée par l'égyptologue italien Ernesto Schiaparelli dans les années 1891-18927 . L'authenticité de ce poignard est toutefois incertaine : la lame, notamment, présente un aspect singulier pour un poignard du Nouvel Empire et se rapproche plutôt d'exemplaires datés par leur contexte de découverte de la Deuxième Période intermédiaire voire du Moyen Empire ${ }^{8}$. De plus, les épaules de la lame ne sont pas entièrement couvertes par la poignée en bois, fait assez inhabituel ; la poignée ellemême est atypique puisque son pommeau figure deux têtes de faucons opposées, fortement stylisées, un motif connu dans l'iconographie royale égyptienne mais très rarement attesté sur de véritables poignards ${ }^{9}$. En admettant néanmoins son caractère antique, le poignard de Florence peut difficilement être rapproché de nos fragments de fourreaux: passé le problème de sa provenance précise qui reste inconnue, ses dimensions ne semblent pas correspondre ${ }^{10}$. Rien n'indique par ailleurs que le cartouche signale que nous avons affaire à un objet royal : on connaît de nombreuses armes épigraphiées où l'inscription se limite à un cartouche accompagné parfois de quelques qualificatifs, mais qui relèvent manifestement de la sphère des particuliers, dont on peut penser qu'ils utilisaient le nom du roi pour ses vertus apotropaïques tout en marquant ainsi leur allégeance ou en insistant sur le lien privilégié qu'ils entretenaient avec leur souverain ${ }^{11}$. 
Fig. 3

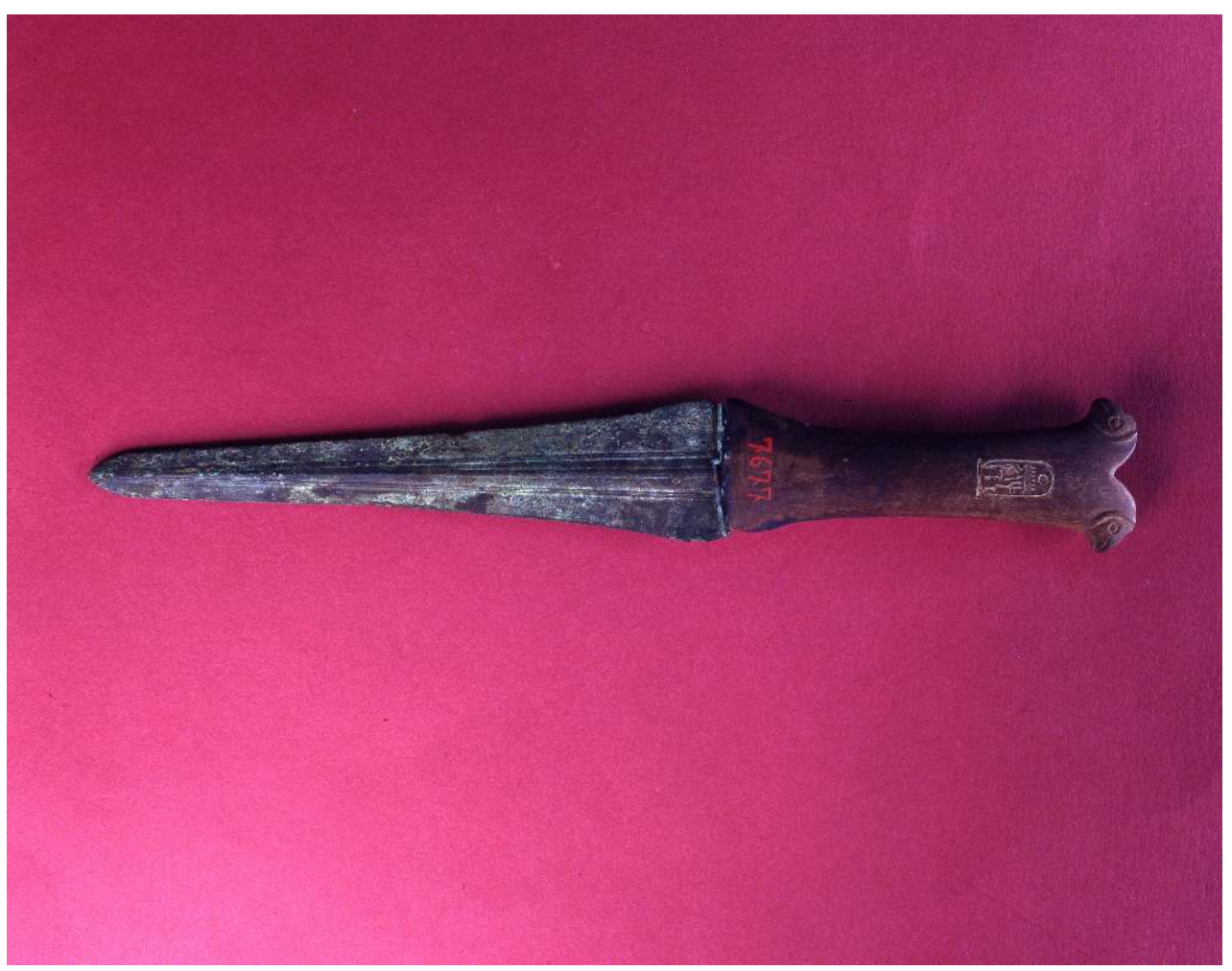

Poignard de Thoutmosis IV - Museo Egizio, Florence, inv. nº 7677

Su concessione della Soprintendenza Archeologia, Belle Arti e Paesaggio per la città metropolitana di Firenze e per le province di Pistoia e Prato

8 L'aspect général et les dimensions des fragments CG 46114 et CG 46115 rappellent en revanche un type de poignard à pommeau piriforme bien connu de l'archéologie soudanaise et de l'égyptologie, dit «type de Kerma », du nom du lieu de provenance principal de ces objets ${ }^{12}$. C'est en effet durant les fouilles conjointes du Museum of Fine Arts de Boston et de l'Université de Harvard, menées par George A. Reisner au début du siècle dernier, que près de 130 poignards de ce type ont été trouvés, souvent glissés dans un fourreau en cuir accroché à la ceinture des défunts de la nécropole de Kerma, complets ou réduits à l'état d'un simple pommeau ou d'une lame isolée ${ }^{13}$. Ces poignards présentent un pommeau de forme allongée, qualifié de "trapézoïdal » ou " piriforme » car il s'évase dans sa partie supérieure. Ce pommeau, le plus souvent en ivoire, est attaché au reste de la poignée, en bois, en alliage cuivreux ou en écaille de tortue, au moyen de rivets généralement en cuivre. La lame est fixée à la poignée également à l'aide de rivets; elle est en bronze ou en cuivre ${ }^{14}$. Les poignards de Kerma sont de tailles variables : les plus petits font environ $25 \mathrm{~cm}$ de longueur, les plus grands atteignent $56 \mathrm{~cm}$. Le pommeau mesure la plupart du temps entre 5 et $5,5 \mathrm{~cm}$ de largeur, la lame $3 \mathrm{~cm}$ de largeur au niveau des épaules.

De nombreuses armes de ce type ont été trouvées dans leur fourreau en cuir, euxmêmes parfois bien conservés ${ }^{15}$. Dans certains cas, l'étui ne protège que la lame; dans d'autres, il recouvre également le pommeau piriforme (fig. 4). Les fourreaux complets présentent toujours une couture centrale sur toute leur longueur. Un rapprochement entre ces fourreaux de poignards de «type Kerma » et les objets en cuir de la tombe de Thoutmosis IV décrits précédemment paraît pertinent, et cohérent en matière de 
dimensions: le premier serait la partie supérieure d'un fourreau, protégeant le pommeau piriforme d'un poignard kermien, alors que le second pourrait être le fourreau complet limité à la lame d'un poignard du même type. On admettra que déposer des fourreaux vides dans une tombe royale serait une pratique pour le moins étrange et que par conséquent ces fragments d'étuis témoignent de la présence d'au moins deux poignards dans le mobilier funéraire de Thoutmosis IV, en partant du principe bien sûr que ces poignards n'aient pas été introduits dans la KV 43 à une période postérieure à celle de l'inhumation du roi. Principalement en métal, ces poignards furent sans doute dérobés par des pillards et appartenaient à une panoplie plus conséquente destinée à accompagner Thoutmosis IV dans l'au-delà, dont seuls quelques vestiges sont conservés ${ }^{16}$. Si l'on admet que ces poignards étaient bien de «type Kerma ", reste à proposer une explication plausible à la présence de ces armes nubiennes dans la tombe d'un pharaon égyptien du Nouvel Empire.

Fig. 4

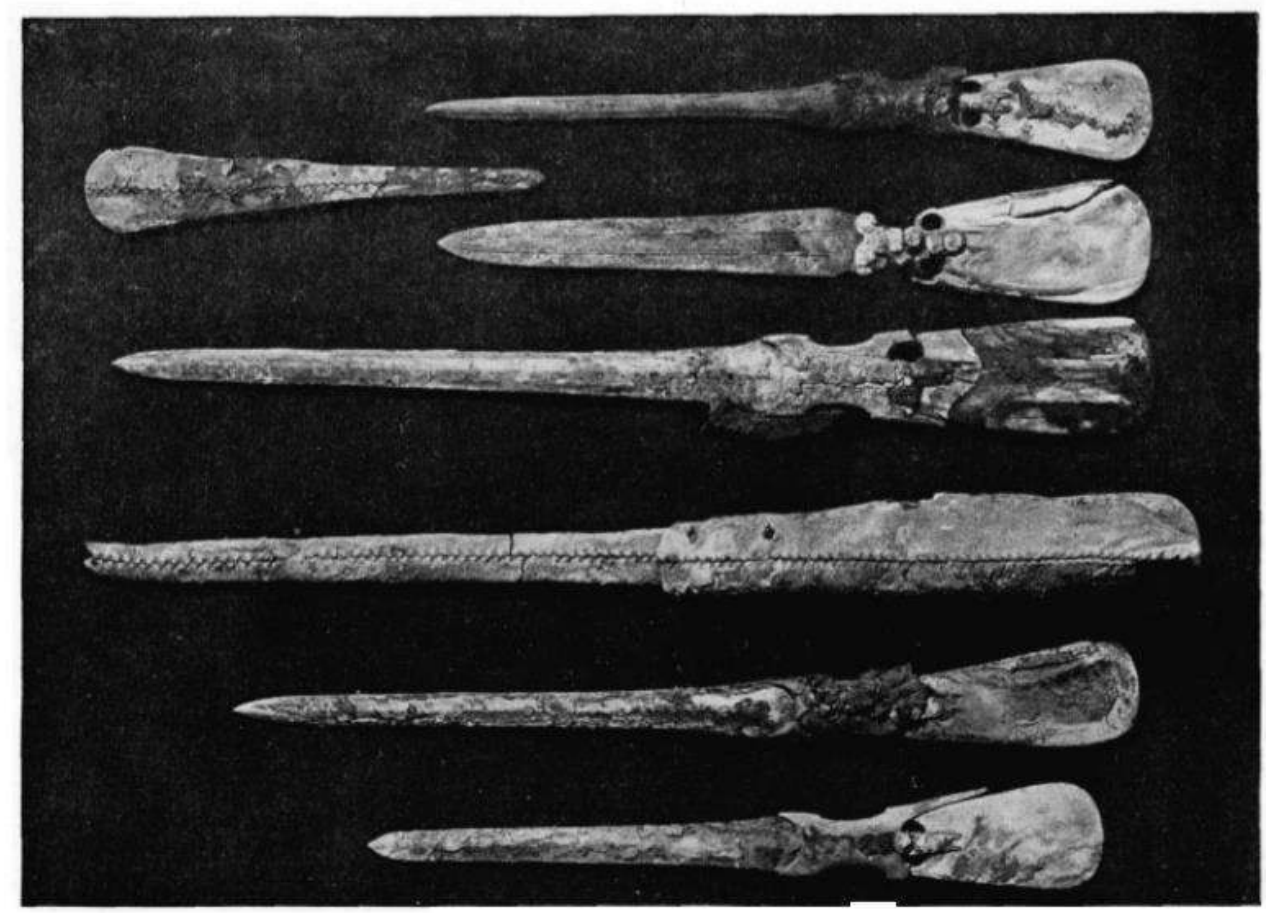

Poignards et fourreaux découverts à Kerma - D'après G. A. Reisner, « Accessions to the Egyptian Collections during $1914 »$, BMFA 13, 1915, p. 80, fig. 14

Car il s'agit bien d'armes "nubiennes", c'est-à-dire culturellement associées à la Nubie $^{17}$ : les poignards de «type Kerma», malgré leur morphologie assez proche des fameux poignards «à lunules » de l'Égypte du Moyen Empire ${ }^{18}$, présentent des caractéristiques qui leur sont propres et facilement reconnaissables, en particulier le pommeau piriforme encadré de deux «bras » en « V » et la lame longue et effilée. La très grande majorité des objets de ce corpus provient de Kerma ou de quelques autres nécropoles nubiennes, et force est de constater que l'on a affaire ici à un marqueur culturel nubien perçu comme tel par les Égyptiens eux-mêmes, ce qu'a parfaitement démontré Susanne Petschel: les deux seules attestations de ce type d'arme dans l'art égyptien, datées respectivement des règnes de Thoutmosis IV ou Amenhotep III et de Toutânkhamon, montrent un Nubien identifiable à la fois par la couleur de sa peau, les 
traits de son visage et sa coiffure, portant un poignard de ce type à la ceinture et à chaque fois dans une attitude de soumission vis-à-vis du pouvoir pharaonique ${ }^{19}$.

Un seul poignard de «type Kerma » a en fait été retrouvé sur le territoire égyptien, en 1822 dans la région thébaine, enchevêtré dans les bandelettes d'une momie d'homme, si l'on en croit l'inventeur de l'objet Giuseppe Passalacqua ${ }^{20}$. Aujourd'hui conservé à l'Ägyptisches Museum und Papyrussammlung de Berlin où il porte le numéro d'inventaire ÄMP $2053^{21}$, ce poignard habituellement daté de la XVIII ${ }^{e}$ dynastie nous est parvenu dans un état remarquable, sans doute grâce à la protection du fourreau de cuir qui l'enveloppait. Si l'on sait peu de choses sur cet objet ${ }^{22}$, il est certain qu'il s'agit d'un exemplaire particulièrement précieux, d'un bien de prestige de grande qualité, comme en témoigne la présence de rivets en or. Le défunt l'a-t-il emporté avec lui dans la tombe pour marquer son origine nubienne ? Ou bien s'agit-il au contraire d'un souvenir rapporté par un haut fonctionnaire égyptien ayant mené une partie de sa carrière en Nubie, voire d'un cadeau diplomatique qu'il y aurait reçu au cours de l'exercice de sa charge? Rien ne permet de trancher, mais les questions soulevées par le poignard de l'ancienne collection Passalacqua rejoignent celles que posent les fourreaux de la KV 43.

Pourquoi trouverait-t-on ce type de poignard, un marqueur culturel et social nubien, dans la tombe d'un roi égyptien de la XVIII dynastie? Plusieurs possibilités sont envisageables.

13 La première, sans doute la plus probable, est celle du cadeau diplomatique, voire $d u$ simple tribut, apporté au roi d'Égypte par ses vassaux de Nubie. On trouve ainsi fréquemment des poignards au sein des présents apportés par les peuples étrangers situés au nord de l'Égypte. Il s'agit sans aucun doute de biens de prestige, en matériaux précieux, témoignant du savoir-faire des artisans de leurs pays d'origine, et destinés autant à impressionner Pharaon qu'à l'honorer en se montrant dignes de son rang. La pratique du cadeau diplomatique, qui implique qu'un souverain offre de riches présents directement à l'un de ses semblables, est bien attestée en Égypte et au Proche-Orient, et ce dès le Moyen Empire ${ }^{23}$. Au Nouvel Empire, l'exemple le plus célèbre de ce type d'échange est bien entendu la dague à lame de fer retrouvée dans la tombe de Toutânkhamon ${ }^{24}$, dont plusieurs auteurs ont relevé la correspondance frappante avec des poignards décrits dans certaines lettres d'Amarna, listant les biens envoyés par le roi du Mitanni à son homologue égyptien ${ }^{25}$. Un poignard retrouvé dans la tombe d'Âhhotep et inscrit au nom du roi Ahmôsis, dont certaines caractéristiques ont été interprétées comme étant d'origine étrangère, probablement crétoise ou mycénienne ${ }^{26}$, pourrait également se rattacher au cadeau diplomatique - pratique qui perdure dans d'autres civilisations aux époques modernes, particulièrement en ce qui concerne les armes $^{27}$. De riches armes de prestiges offertes par des chefs nubiens soumis ou désireux de plaire à Thoutmosis IV et qui en portaient eux-mêmes à leur ceinture, voilà un scénario qui pourrait bien expliquer la présence de ces poignards de Kerma dans la tombe du roi : les matériaux précieux employés dans leur fabrication, que l'exemplaire de la collection Passalacqua nous permet d'imaginer, ont évidemment incité les pillards à s'en emparer dès l'Antiquité.

14 La question d'un éventuel trophée militaire mérite également d'être soulevée. Les textes dits « historiques », narrant les campagnes syriennes ou nubiennes des pharaons du Nouvel Empire, mentionnent fréquemment des armes au sein des listes de butins rapportés pour alimenter les trésors du roi et des temples du Double Pays ${ }^{28}$. Il s'agit 
souvent de grandes quantités d'armes et l'Égypte profitait sans doute de ses victoires pour remplir ses arsenaux en vue des prochaines batailles, en plus de la valeur brute que pouvaient représenter ces objets en grande partie en bronze, dans un monde où le contrôle des routes de l'étain était au cœur des conflits entre grandes puissances ${ }^{29}$. Si, à ma connaissance, les poignards sont absents des listes de butin du Nouvel Empire, des poignards en bronze, or, argent ou ivoire sont évoqués à trois reprises dans les Annales d'Amenemhat $\mathrm{II}^{30}$. On trouve parfois dans les textes la mention d'armes manifestement revêtues d'une valeur particulière : dérobées à un chef ennemi à l'issue du combat ${ }^{31}$, elles devaient constituer des trophées, probablement exposés comme tels et intégrés à l'inévitable opération de propagande qui suivait les victoires de Pharaon et servait à humilier encore un peu plus les Neuf Arcs. Dans un registre assez proche, certains textes autobiographiques bien connus relatent le butin personnel dont des "héros " égyptiens s'emparaient et qui leur permettait ensuite d'être distingués par le roi et d'obtenir «l'or de la récompense ${ }^{32}$ ». Mais c'est un document du début de la XVIII dynastie qui se rapproche le plus de la notion moderne de "trophée » : une pointe de lance en bronze conservée au musée Pouchkine de $\mathrm{Moscou}^{33}$ et dont l'inscription précise qu'elle a été rapportée à la suite de la victoire des troupes thébaines d'Ahmôsis sur les Hyksôs dans leur capitale d'Avaris ${ }^{34}$. Les activités militaires de Thoutmosis IV en Nubie, si elles restent limitées par rapport à d'autres règnes, sont relativement bien documentées ${ }^{35}$, et il est parfaitement plausible que le roi ait pris de riches poignards à quelques chefs nubiens vaincus lors de ses expéditions méridionales.

Les tombes de particuliers ont livré de nombreux poignards, sous forme de modèles ou bien représentés au sein des frises d'objets peints sur les cercueils du Moyen Empire ${ }^{36}$. Si l'on en trouve régulièrement dans des tombes ayant appartenu à d'anciens militaires, il faut remarquer qu'en Égypte, cette arme de "coup de grâce », de faible portée et utilisable seulement en combat de proximité, est souvent la marque d'un certain rang social : un objet de prestige, qui peut être en matériaux précieux, richement décoré et inscrit, presque un bijou dont se parent également certaines femmes ${ }^{37}$. On est d'ailleurs en droit de se demander s'il ne faut pas établir un lien entre ce rôle de marqueur social et le signe hiéroglyphique T8 et ses variantes de la Sign-List de Gardiner, fréquemment utilisé pour noter le premier des ordinaux en égyptien ancien, $t p(y)^{38}$. Peut-on aller jusqu'à voir dans le poignard une sorte de « marqueur hiéroglyphique » du statut social élevé du défunt? Notons que l'emploi de tp comme désignation archaïque du poignard, envisagé par A. H. Gardiner ${ }^{39}$ et qui aurait été remplacé par le mot $b 3 g s w / m 3 g s w^{40}$, n'est confirmé a priori que par la légende d'un cercueil du Moyen Empire provenant de la région thébaine ${ }^{41}$. La présence de modèles dans certaines tombes semble indiquer qu'on accordait également à ce type d'objets une valeur symbolique ou rituelle dont la compréhension nous échappe encore très largement ${ }^{42}$, mais dont il faut sans doute aussi percevoir une manifestation dans certaines «amulettes-poignards" datées de Basse Époque ${ }^{43}$.

Thoutmosis IV n'est pas le seul roi à s'être fait enterrer accompagné de poignards ${ }^{44}$. À l'époque historique ${ }^{45}$, cette pratique est attestée dès le Moyen Empire ${ }^{46}$, mais aussi chez d'autres rois de la XVIII dynastie tels qu'Amenhotep I $^{47}$ ou Toutânkhamon ${ }^{48}$, bien que les armes semblent à chaque fois très différentes. Le poignard, bien plus qu'une arme de combat, est un objet personnel, que l'on offre ou que l'on transmet, lorsqu'on ne l'emporte pas avec soi dans la tombe; un marqueur social et culturel, qui en dit beaucoup sur son propriétaire et le monde qui l'entoure, en Égypte ou ailleurs. Si les simples «empreintes» de cuir que sont les fourreaux de Thoutmosis IV permettent 
d'aborder de nombreuses problématiques et de formuler diverses propositions, même si celles-ci restent cantonnées au domaine de l'hypothèse, elles donnent un aperçu de l'intérêt que peut avoir l'étude des poignards ou plus largement de l'armement ancien, dans des domaines variés, de l'histoire des techniques à la lexicographie.

\section{NOTES}

1. Sur ce sujet, voir l'article de Nozomu Kawai, «Development of the Burial Assemblage of the Eighteenth Dynasty Royal Tombs », Orient, vol. 35, 2000, pp. 35-59.

2. Sur Thoutmosis IV et son règne en général, voir notamment l'ouvrage de Betsy M. Bryan, The Reign of Thutmose IV, Baltimore et Londres, The Johns Hopkins Press, 1991.

3. Howard Carter, Percy E. Newberry, Catalogue général des antiquités égyptiennes du Musée du Caire,

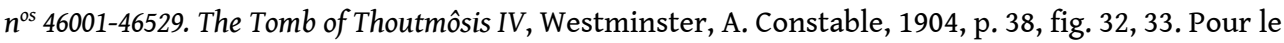
mobilier funéraire de Thoutmosis IV, voir aussi les compléments apportés par B. M. Bryan, op. cit. note 2, pp. 208-210; sur la KV 43 en général, cf. Nicholas Reeves, Richard H. Wilkinson, The Complete Valley of the Kings. Tombs and Treasures of Egypt's Greatest Pharaohs, Londres, Thames \& Hudson, 1996, pp. 105-108.

4. Estimation d'après photographie.

5. Ce motif décoratif se retrouve assez souvent sur les lames de poignards. Il est très fréquent en particulier sur certaines lames larges à double tranchant convexe qui forment un sous-corpus bien connu d'objets situés à la frontière entre les couteaux et les poignards : un canard prenant son envol est souvent représenté au-dessus de l'ombelle de papyrus, et on connaît même un cas comportant une représentation de bouquetin (Cleveland Museum of Art, inv. $n^{\circ}$ 1983.212). On retrouve l'ombelle de papyrus sur des représentations de ces lames visibles dans la tombe de Qenamon, cf. Norman de Garis Davies, The Tomb of Ken-Amun at Thebes, New York, Metropolitan Museum of Art, PMMA 5, 1930, pl. XVI-XVIII. Le sens de ces décors et la nature de ces objets restent à étudier de manière plus approfondie: sur ce type de lames, cf. récemment Susanne Petschel, Den Dolch betreffend. Typologie der Stichwaffen in Ägypten von der prädynastischen Zeit bis zur 3. Zwischenzeit, Wiesbaden, Harrassowitz Verlag, Philipikka_36, 2011, pp. 267-269 et R. Pietri, Poignards, épées et harpés: de la fabrication au cadeau diplomatique, mémoire de recherche inédit de l'École du Louvre, 2 vol., Paris, 2011, vol. 1, pp. 82-87.

6. Inv. $n^{\circ} 7677$. À noter que la lecture du cartouche pose des difficultés, alors qu'il s'agit du principal indice pour la datation de l'objet, cf. note suivante. Je lis personnellement - et d'après photographie - «Mn-Hpr.w-R'-wsr », une variante a priori inconnue par ailleurs de «Mn-Hpr.w-R`» , le nom de couronnement de Thoutmosis IV (cf. Jurgen von Beckerath, Handbuch der ägyptischen Königsnamen (2 éd.), Mainz, Münchner Ägyptologische Studien (MÄS) 49, 1999 [1984], pp. 138-140).

7. Sur ce poignard, cf. cat. d'exp., La Battaglia di Qadesh. Ramesses II contro gli Ittiti per la conquista della Siria, sous la direction de Maria Cristina Guidotti et Franca Pecchioli Daddi, Florence, Musée égyptien, 6 juin-8 décembre 2002, Florence, Sillabe, 2002, p. 71, nº 27 et M. C. Guidotti (éd.), Il carro e le armi del Museo Egizio di Firenze, Florence, Giunti, Materiali del Museo Egizio di Firenze 2 (MAAT) 2, 2002, p. 56, n 22. Voir aussi la notice consacrée à l'objet sur le site du Global Egyptian Museum, où le cartouche est lu «Mn-Hpr-R'-wsr» et attribué à Thoutmosis III : www.globalegyptianmuseum.org/record.aspx?id=10114 [27/12/2015]. 
8. Il pourrait s'agir d'une lame du Moyen Empire montée sur un manche plus tardif, voire un faux éventuel. Ce type de montage moderne est attesté dans d'autres cas, cf.par exemple S. Petschel, op. cit. note 5, p. 528, $\mathrm{n}^{\circ} 299$. Pour un objet assez proche du poignard de Florence sur le plan formel mais anépigraphe, avec notamment deux protubérances au niveau du pommeau mais cette fois-ci non-incisées, voir un poignard conservé au Fitzwilliam Museum de Cambridge, inv. $\mathrm{n}^{\circ}$ E.377.1932, cf. S. Petschel, op.cit. note 5, p. 490, $\mathrm{n}^{\circ} 230$; notice en ligne sur le site du Fitzwilliam Museum : data.fitzmuseum.cam.ac.uk/id/object/53123 [28/12/2015]. Il s'agit là aussi probablement d'un assemblage avec un manche secondaire, mais peut-être antique. Un poignard au manche inscrit au nom de Ramsès $\mathrm{V}$, et au pommeau décoré d'une tête hathorique, mais manifestement fixé à une lame moderne ou provenant d'un autre objet, est passé en vente chez Christie's le 9 décembre 2010 (vente $\mathrm{n}^{\circ} 2364$, lot $\mathrm{n}^{\circ}$ 14, ancienne collection M. Palevsky, cf. www.christies.com/lotfinder/ancient-art-antiquities/an-egyptian-wood-and-bronze-daggernew-5385320-details.aspx?from=salesummary\&intObjectID=5385320\&sid=8b67b1c3a81c-4f49-9bd6-98c23d016810 [28/12/2015]). Cette arme est atypique et son authenticité a de quoi être interrogée.

9. Sur ce type de poignards avec pommeau à têtes de faucons, cf. S. Petschel, op.cit. note 5, pp. 224-230 (type VIII) ; R. Pietri, op. cit. note 5, pp. 77-81. Ce motif est attesté pour la première fois sur une statuette du Louvre attribuée à Amenemhat III, portant le numéro d'inventaire N 464, cf. Élisabeth Delange, Catalogue des statues égyptiennes du Moyen Empire : 2060-1560 av. J.-C., musée du Louvre, Paris, Réunion des musées nationaux (RMN), 1987, pp.33-35 mais d'après l'auteur, il s'agirait d'une gravure secondaire : cette hypothèse reste à démontrer. Le motif - et ses variantes avec deux têtes opposées, avec ou sans disque solaire - est ensuite présent surtout dans la statuaire royale du Nouvel Empire, et n'apparaît de manière certaine sur une arme que sous la XXI ${ }^{e}$ dynastie, avec le manche de l'épée de Psousénnès I ${ }^{\text {er }}$ trouvé dans la NRT III à Tanis et conservé au Musée égyptien du Caire (JE 85854) : cf. en dernier lieu sur cet objet et sa découverte S. Petschel, op. cit. note 5, p. 484, $\mathrm{n}^{\circ} 220$ et Patrice Le Guilloux, Le mobilier funéraire de Psousennès $I^{e r}$, Arles, Actes Sud, Cahier de Tanis n 2, 2010, p. 94, nº 35 et pp. 262, 263. D'autres poignards dotés de cet élément thériomorphe, dont l'authenticité est toutefois suspecte, sont connus en plus de l'objet de Florence: Carnegie Museum of Natural History, Pittsburgh, inv. $\mathrm{n}^{\circ}$ 9074-2464 (S. Petschel, op.cit. note 5, pp. 485, $\mathrm{n}^{\circ} 221$ ); poignard de l'ancienne collection Rustafjaell, Catalogue of the Remaining Part of the Valuable Collection of Antiquities formed by Robert de Rustafjaell, Londres, Sotheby, Wilkinson \& Hodge, 1913, pl. XXXIII (peut-être un assemblage moderne). Malgré la synthèse proposée en 2011 par Susanne Petschel, une étude complète reste à faire sur ce motif, à la fois dans l'art et dans le domaine de l'hoplologie à proprement parler.

10. Longueur de $33 \mathrm{~cm}$; largeur max. d'environ $4,5 \mathrm{~cm}$ (d'après photographie).

11. Voir aussi sur le sujet mes remarques dans un précédent article de cette même revue: R. Pietri, « À propos d'une lame de poignard inscrite au nom d'un roi Menkheperrê : notes sur un objet singulier conservé au Louvre (AF 13446) », Cahiers de l'École du Louvre 1, 2012, en particulier p. $7, \mathrm{n}^{\circ} 29$ et pp. $8,9, \mathrm{n}^{\circ} 41$.

12. Sur les poignards de Kerma, cf. S. Petschel, op. cit. note 5, pp. 170-180 et 450, 451 (type IV) et notes suivantes. Voir aussi l'étude de Henriette Hafsaas-Tsakos, "Edges of bronze and expressions of masculinity: the emergence of a warrior class in Sudan », Antiquity 87, 2013, pp. 79-91.

13. Cf. George A. Reisner, Excavations at Kerma, I-III, Cambridge Mass., Peabody Museum of Harvard University, Harvard African Studies (HAS) V, 1923, passim ; idem, Excavations at Kerma, IVV, Cambridge Mass., Peabody Museum of Harvard University, HAS VI, 1923, passim.

14. Les poignards de Kerma ont fait l'objet d'un certain nombre d'analyses visant à déterminer la composition chimique de leurs lames, et parfois des rivets impliqués dans le système de fixation lame-manche, cf. Dows Dunham, « Notes on Copper-Bronze in the Middle Kingdom », The Journal of Egyptian Archaeology (JEA) 29, 1943, pp.60-62; Denise Thomas-Goorieckx, René Lefève, 
«Examen et traitement d'une dague en bronze», Kush 8, 1960, pp. 266, 267; Susanne M. M. Young, «Archaeometric Analysis of Copper Swords from Kerma (Nubia)», dans Lech Krzyzaniak, Karla Kroeper, Michal Kobusiewicz (éd.), Interregional Contacts in the Later Prehistory of Northeastern Africa, Poznan, Muzeum Archeologiczne w Poznaniu, Studies in African Archaeology (SAA) 5, 1996, pp. 475-490. Voir aussi à ce propos le récent article de Faïza Drici, "The chaîne opératoire of Bronze Working in Ancient Sudan: An Attempt at Reconstituting the Manufacture of Kushite Weapons ", Dotawo 3, 2016, pp. 9-28, http://digitalcommons.fairfield.edu/djns/vol3/ iss1/1. Il ressort de ces analyses que si le bronze est bien attesté, le cuivre simplement arsénié et/ ou écroui est également très fréquent.

15. G. A. Reisner, "Accessions to the Egyptian Collections during 1914 », Museum of Fine Arts Bulletin (BMFA) 13, 1915, p. 80, fig. 14.

16. Voir H. Carter, P. E. Newberry, op. cit. note 3, pp. 24-38. Sur le principal élément de cette panoplie, le caisson de char trouvé dans la tombe de Thoutmosis IV, cf. le nouvel article de référence d'Amy M. Calvert, «Vehicle of the Sun: The Royal Chariot in the New Kingdom », dans André J. Veldmeijer, Salima Ikram (éd.), Chasing Chariots. Proceedings of the First International Chariot Conference (Cairo 2012), Leiden, Sidestone Press, 2013, pp. 45-71. Les étiquettes hiératiques trouvées dans la tombe mentionnent certains objets qui complétaient l'arsenal du roi, cf. H. Carter, P. E. Newberry, op. cit. note 3, pp. 39, 40: elles feront l'objet d'un article ultérieur actuellement en préparation («Notes sur le mobilier funéraire de Thoutmosis IV. §2 - Les étiquettes hiératiques de la KV $43 »)$.

17. La question a divisé les spécialistes, certains voyant dans Kerma une colonie égyptienne et d'autres une culture autochtone, le poignard étant un argument parmi d'autres : cf. entre autres Jean Vercoutter, "A Dagger from Kerma», Kush 8, 1960, p. 265 ; Fritz Hintze, "Das KermaProblem », Zeitschrift für ägyptische Sprache und Altertumskunde (ZÄS) 91, 1964, p. 84.

18. Sur les poignards à lunules, datés principalement du Moyen Empire mais qui connaissent des variantes jusqu'au début du Nouvel Empire, cf. S. Petschel, op.cit. note 5, pp. 106-169 et pp. 362-448 (type III) ; Dietrich Raue et al., «Report on the $35^{\text {th }}$ Season of Excavation and Restoration on the Island of Elephantine ", Annales du service des antiquités de l'Égypte (ASAE) 82, 2008, pp. 212, 213. En Égypte, le type «à lunules » semble avoir connu une évolution formelle qui aboutit à des poignards dont le pommeau prend de plus en plus d'importance à mesure que la taille du manche diminue et que la lame triangulaire s'affine et adopte deux tranchants convexes. Les meilleurs exemples de ce dernier stade sont sans doute les poignards de Kamosis (Cabinet des Médailles, Bibliothèque royale de Belgique) et d'Ahhotep (Musée égyptien du Caire, JE 4668), voir en dernier lieu S. Petschel, op. cit. note 5, pp. 410, 411, $\mathrm{n}^{\text {os }}$ 88-89 et Céline Ben Amar, « The Dagger of Pharaoh Kamose, the Oldest Glory of the Royal Library of Belgium », In Monte Artium 5, 2012, pp. 45-67.

19. Cf. S. Petschel, op. cit. note 5, pp. 175, 176 : peinture murale du scribe des recrues Horemheb (TT 78) ; repose-pieds de Toutânkhamon (Musée égyptien du Caire, JE 62047).

20. Giuseppe Passalacqua, Catalogue raisonné et historique des antiquités découvertes en Égypte, Paris, Galerie d'antiquités égyptiennes, 1826, p. 28, nº 550 et p. 155. Sur la vie et la carrière de Giuseppe Passalacqua, cf. Morris L. Bierbrier (éd.), Who Was Who in Egyptology (4 éd.), Londres, Egypt Exploration Society, 2012 [1951], p. 418.

21. S. Petschel, op. cit. note 5, p. 450, $\mathrm{n}^{\circ} 166$ (avec bibliographie).

22. Les circonstances de la découverte et le lieu d'inhumation de la momie restent à ce jour assez flous, malgré les précisions de Passalacqua: "cette momie si riche en objets marquans, et déposée dans un cercueil couvert de belles peintures, n'était pas placée dans un tombeau creusé dans la roche. Elle fut découverte dans la nécropolis de Thèbes, sous cinq à six pieds de débris de pierres, provenant des anciens travaux et excavations environnantes » (cf. G. Passalacqua, op. cit. note 20 , p. 155). L'hypothèse de Passalacqua, qui voit dans cette inhumation peu orthodoxe un signe de la vindicte populaire souhaitant punir à titre posthume un criminel, «ainsi que le 
poignard [...] qu'on trouva sur lui paraîtrait l'indiquer » (G. Passalacqua, op. cit. note 20, p. 204), est bien sûr à abandonner : il faut plutôt y voir une tombe très perturbée par les fouilles de l'époque et dont le contexte reste encore à reconstituer. On notera que la lame du poignard a été analysée au XIX ${ }^{\mathrm{e}}$ siècle par Louis-Nicolas Vauquelin, membre de l'Institut de France, qui donne les indications suivantes : «Le métal enlevé de dessus ce poignard n'est que du cuivre mêlé d'une matière résineuse qui, sans doute, lui servait de vernis, en même temps qu'elle le défendait de la rouille. Il est possible que ce cuivre contienne un peu d'étain; mais je n'ai pu le constater sur la très-petite quantité qui m'en a été remise. » (G. Passalacqua, op. cit. note 20, p. 238).

23. Cf. par exemple cat. d'exp, Beyond Babylon. Art, Trade, and Diplomacy in the Second Millennium B.C., sous la direction de Joan Aruz, Kim Benzel, Jean Evans, New York, Metropolitan Museum of Art, 18 novembre 2008-15 mars 2009, New York, Metropolitan Museum of Art, 2008, pp. 38, 39, $\mathrm{n}^{\circ}$ 12.

24. Musée égyptien du Caire, JE 61585 A.

25. Sur ce sujet, voir en dernier lieu Marc Gabolde, Toutânkhamon, Paris, Pygmalion, 2015, pp. 368, 369. La lame de fer de ce poignard a été analysée récemment et il a été démontré qu'il s'agissait de fer météoritique, cf. Daniela Comelli et al., « The Meteoritic Origin of Tutankhamun's Iron Blade ", Meteoritics and Planetary Science (MPS) 51/7, 2016, pp. 1-9; voir néanmoins contra une analyse plus ancienne, cf. Fatma M. Helmi, Kamal Barakat, « Micro Analysis of Tutankhamun's Dagger », dans Faisal A. Esmael, Zahi Hawass (éd.), Proceedings of the First International Congress on Ancient Egyptian Mining and Metallurgy and Conservation of Metallic Artifacts (10-12 April 1995), Le Caire, Ministry of Culture, Supreme Council of Antiquities, 1996, pp. 287, 288. Sur les poignards de Toutânkhamon, cf. aussi S. Petschel, op. cit. note 5, pp. 482, 483, n ${ }^{\text {os }} 218,219$ (avec bibliographie).

26. Musée égyptien du Caire, CG 52658, cf. S. Petschel, op. cit. note 5, p. 486, $\mathrm{n}^{\circ} 222$. Voir aussi le poignard du Louvre inscrit au nom d'Ahmôsis, cf. Nathalie Couton-Perche, Dominique Robcis, Marc Aucouturier, Juliette Langlois, "Une dague au nom d'Âhmosis : une réhabilitation?", Memnonia 22, 2011, pp. 135-147, pl. XXV-XL.

27. Cat. d'exp., L'épée. Usages, mythes et symboles, sous la direction de Almudena Biasco, Fabrice Cognot, Christine Duvauchelle, Michel Huynh et Iaroslav Lebedynsky, Paris, Musée de ClunyMusée national du Moyen Âge, 28 avril-28 septembre 2011, Paris, RMN, 2011, p. 77 (fig. 84).

28. Sur ce sujet, cf. Robert G. Morkot, Historical Dictionary of Ancient Egyptian Warfare, Lanham, Md, et Oxford, Scarecrow Press, 2003, pp. 24-27 ; idem, « War and the Economy: the International "arms trade" in the Late Bronze Age and after ", dans Thomas Schneider, Kasia Szpakowska (éd.), Egyptian Stories: A British Egyptological Tribute to Alan B. Lloyd on the occasion of his Retirement, Münster, Ugarit Verlag, Alter Orient und Altes Testament (AOAT) 347, 2007, pp. 169-195.

29. Pierre Grandet, Les pharaons du Nouvel Empire, une pensée stratégique (1550-1069 avant J.-C.), Monaco, Éditions du rocher, 2008, pp. 30-49.

30. Sur les fragments des Annales d'Amenemhat II, voir en dernier lieu Hartwig Altenmüller, Zwei Annalenfragmente aus dem frühen Mittleren Reich, Hambourg, Helmut Buske Verlag, Studien zur altägyptischen Kultur (SAK) VIII, 2015, en particulier pp. 67, 73, $\mathrm{n}^{\circ} 8$ (fragment M, col. $\mathrm{x}+16$, b3gsw

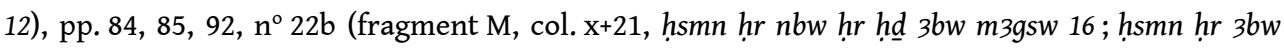
m3gsw 21 (?)) pour les passages évoqués ci-dessus.

31. Par exemple au prince de Megiddo sous Thoutmosis III, cf. Urk. IV, 663, 14-15 (wrry.t nfr.t b3k= tj m nbw n(y) wr n(y) [Mktj]) ; 664, 4 (hsmn mss nfr n(y) ‘ḩ3n(y) wr n(y) Mk[tj]).

32. L'or de la récompense pouvait prendre des formes variables: outre le célèbre collier-šbjw, différents objets en or, bijoux mais aussi armes, étaient distribués aux combattants méritants, ce qui n'est pas sans rappeler entre autres les " armes d'honneur » offertes à certains soldats de la Révolution puis de l'Empire (voir par exemple Thierry Lentz, Le Grand Consulat, 1799-1804, Paris, Fayard, 2014 [1999], pp.570-575, avec bibliographie). Ce phénomène est assez bien renseigné pour l'Égypte pharaonique, et ce au moins depuis le Moyen Empire avec la stèle de Khousobek, 
conservée au Manchester Museum, inv. $\mathrm{n}^{\circ}$ 3306, datée de la XII ${ }^{\mathrm{e}}$ dynastie, règne de Sésostris III. Sur ce document, cf. Thomas Eric Peet, The Stela of Sebek-Khu: The Earliest Record of an Egyptian Campaign in Asia, Manchester, Sherratt \& Hughes, 1914 et cat. d'exp., Pharao siegt immer: Krieg und Frieden im alten Ägypten, sous la direction de S. Petschel et Martin von Falck, Hamm, Gustav Lübcke Museum, 21 mars-31 octobre 2004, Bonn, Kettler, 2004, pp. 158, 159, n 155 , mais aussi récemment cat. d'exp., Sésostris III, pharaon de légende, sous la direction de Fleur Morfoisse et Guillemette Andreu-Lanoë, Lille, Palais des Beaux-Arts, 10 octobre 2014-26 janvier 2015, Gand, Snoeck, 2014, pp. 148, 149, fig. 7 et 286, nº 195 et cat. d'exp., Ancient Egypt Transformed: The Middle Kingdom, sous la direction de Adela Oppenheim, Dorothea Arnold, Dieter Arnold, Kei Yamamoto, New York, Metropolitan Museum of Art, 12 octobre 2014-24 janvier 2015, New York, Metropolitan Museum of Art, 2015, p. 166, $n^{\circ}$ 99. Le passage de la stèle de Khousobek, dont la fin est de lecture incertaine, indique: ' $h^{\prime} \sim n d \sim n=f n=j s \underline{t} s \quad m \underline{d^{\prime}} m r \underline{d} r . t=j j w n . t h n^{\prime} m(3) q s w$ (= m3gsw) b3k=wm $\underline{d}^{\prime} m$ $h n^{\prime} H^{\prime} w=s n(?)$. Au Nouvel Empire, les différentes versions de l'autobiographie d'Ahmès Pennekhbet évoquent aussi ce type d'armes en matériaux précieux : un poignard en or (= Urk. IV, $38,11-12)$; deux haches en or (= Urk. IV, 39, 1); deux haches en argent (= Urk. IV, 39, 3). Une lame de hache inscrite du British Museum (EA 37447), en alliage cuivreux (6,4 \% d'étain), est aussi à rattacher à ce dossier des "armes d'honneur " pharaoniques, le texte précisant : $r d=w m$ hsw.t $n(y)$-sw.t $n w^{c} w n(y)$ Mry-Jmn Nhm ; sur cet objet, cf. Eva Kühnert-Eggebrecht, Die Axt als Waffe und Werkzeug im alten Ägypten, Berlin, MÄS 15, 1969, pp. 59, 60, 133 et Vivian W. Davies, Catalogue of Egyptian Antiquities in the British Museum, VI. Tools and Weapons, I: Axes, Londres, British Museum Publications, 1987, p. 51, $\mathrm{n}^{\circ} 156$. Enfin, dans la tombe de Qenamon (TT 73), datée du règne d'Amenhotep II, c'est un char donné par faveur du roi qui est mentionné, sans précisions supplémentaires : wrr.yt $r d(w . t) \sim n h m=f m$ hsw.t (= Urk. IV, 1399, 19). Sur l'or de la récompense en général, le lecteur intéressé consultera Susanne Binder, The Gold of Honour in New Kingdom Egypt, Oxford, Aris and Phillips, Australian Center for Egyptology (ACE), Studies 8, 2008, en particulier pp. 74, 75 concernant les exemples liés à l'armement, ainsi que Sonia Focke, " "His Majesty Saw my Valour": Weapons as Rewards for Feats on the Battlefields ", dans Stephen O'Brien, Daniel Boatright (éd.), Warfare and Society in the Ancient Eastern Mediterranean, Oxford, Archaeopress, BAR International Series 2583, 2013, pp.5-18. La question des "armes d'honneur» dans l'Égypte ancienne a également été abordée en relation avec un passage de la stèle du vice-roi Ousersatet qui mentionne une hache en électrum (jqhw $m \underline{d}^{\prime} m$ ) apparemment proverbiale : sur ce texte, cf. en dernier lieu John Coleman Darnell, « The Stela of the Viceroy Usersatet (Boston MFA 25.632), his Shrine at Qasr Ibrim, and the Festival of Nubian Tribute under Amenhotep II ", Égypte nilotique et méditerranéenne (ENIM) 7, 2014, pp. 239-276.

33. Inv. $n^{\circ}$ I.1.a.1762, cf. Oleg Berlev, Svetlana Hodjache, «Objets royaux du musée des Beaux-Arts Pouchkine à Moscou », Chronique d'Égypte (CdE) 52, 1977, pp. 22-29; idem, "A Spearhead of the Pharaoh Ahmose in the Pushkin Museum », Vestnik Drevnej Istorii (VDI) 149, 1979, pp. 82-84 [en russe avec résumé en anglais].

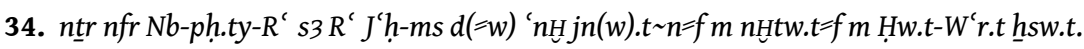

35. Sur ce sujet, cf. par exemple Betsy M. Bryan, "Antecedents to Amenhotep III ", dans David O'Connor, Eric H. Cline (éd.), Amenhotep III: Perspectives on his Reign, Ann Harbor, University of Michigan Press, 1998, pp.54-58. Le principal document du règne concernant les activités militaires du roi en Nubie est la stèle de Konosso, cf. Andrea Klug, Königliche Stelen in der Zeit von Ahmose bis Amenophis III, Turnhout, Brepols Publishers, Monumenta Aegyptic 8, 2002, pp. 345-352 (= Urk. IV, 1545, 1-1548, 6).

36. Cf. S. Petschel, op. cit. note 5, passim. Sur les frises d'objets, voir en particulier Gustave Jéquier, Les frises d'objets des sarcophages du Moyen Empire, Le Caire, Institut français d'archéologie orientale, Mémoires de l'Institut français d'Archéologie orientale (MIFAO) 47, 1921, pp. 195-200.

37. Parmi les cas notables de personnages féminins accompagnés de poignards dans la tombe, outre le cas bien connu de la reine Ahhotep, citons celui de la princesse Ita, cf. S. Petschel, op. cit. 
note 5, p. $358, n^{\circ} 13$ (avec bibliographie). Voir aussi le cas du poignard à pommeau en forme de tête de faucon que l'on a proposé de restituer au sein du mobilier funéraire de la princesse Noubheteptikhered, cf. Sidney H. Aufrère, «Le roi Aouibrê Hor. Essai d'interprétation du matériel découvert par Jacques de Morgan à Dahchour", Le Bulletin de l'Institut français d'archéologie orientale (BIFAO) 101, 2001, p. 32 : l'auteur va peut-être un peu loin lorsqu'il estime que « de tels poignards, au pommeau en forme de tête de faucon, sont communs aux statues de l'époque d'Amenemhat III ", cf. supra note 8, en tout cas dans l'état actuel de la documentation.

38. $W b . V, 279,7$.

39. Alan H. Gardiner, Egyptian Grammar ( $3^{\mathrm{e}}$ éd.), Oxford, Griffith Institute, 1982 [1927] p. 511.

40. Wb. I, 432, 4-5 et II, 33.

41. Cercueil d'un certain Montouhotep, cf. Wb. V, 293, 2 et G. Steindorff, Grabfunde des mittleren Reiches in den königlichen Museen zu Berlin, Berlin, Speman, Mitteilungen aus den orientalischen Sammlungen (MOS) 8, 1896, pp. 28, 29, pl. V (cité par G. Jéquier, op. cit. note 36, p. 200).

42. Le lecteur intéressé par cette question pourra consulter notamment les réflexions récentes sur un sujet assez proche de Cédric Larcher, «Le sens des rites. $§ 1$ - La scène de découpage rituel des armes dans la tombe de Montouherkhepechef à Dra Abou el-Naga », dans Band 44, SAK VI, 2015, pp. 191-214, qui met en valeur à travers l'exemple de l'arc les différents rôles que peuvent assumer les armes déposées dans les tombes, supports de rites ou simples outils mis à disposition du défunt dans l'au-delà.

43. On trouve ainsi le mot b3gsw dans une liste d'amulettes d'époque tardive écrite au verso d'un Livre des Morts, cf. Jean Capart, « Une liste d'amulettes », ZÄS 45, 1908-1909, pp. 14-21.

44. Je ne reviendrai pas ici sur la problématique de l'armement en général en contexte funéraire - royal ou particulier -, qui est d'un grand intérêt mais sort du cadre de cet article. Chaque arme a ses spécificités et mériterait un développement en conséquence avant même de penser à une approche plus synthétique.

45. Sur les poignards de l'époque prédynastique, cf. notamment S. Petschel, op. cit. note 5, pp. 49-55 et 350-353, nos 1-4 ; compléter la bibliographie fournie par Susanne Petschel avec Ulrich Hartung, «Ein Fragment eines verzierten Dolchgriffs aus dem Friedhof $U$ in Abydos (Umm elQaab) », dans Eva M. Engel, Ulrich Hartung, Vera Müller (éd.), Zeichen aus dem Sand. Streiflichter aus Ägyptens Geschichte zu Ehren von Günter Dreyer, Wiesbaden, Harrassowitz Verlag, MENES n ${ }^{\circ} 5$, 2008, pp. 183-194.

46. David Lorand, «Le matériel funéraire de Sésostris I ${ }^{\text {er }}$ conservé au Musée égyptien du Caire », Bulletin de l'Institut français d'archéologie orientale (BIFAO) 111, 2011, p. 268.

47. Fragments de fourreaux conservés au Musée égyptien du Caire, CG 24122, 24122 bis et 24123, cf. Georges Daressy, Catalogue général des antiquités égyptiennes du Musée du Caire, $n^{\text {os }}$ 24001-24990. Fouilles de la Vallée des Rois (1898-1899), Le Caire, Institut français d'archéologie orientale, 1902, pp. 69, 70 et pl. XIX.

48. Cf. supra note 25 .

\section{RÉSUMÉS}

La tombe de Thoutmosis IV (KV 43), fouillée au début du Xx $\mathrm{x}^{\mathrm{e}}$ siècle par Howard Carter, a livré deux fragments de fourreaux en cuir attestant vraisemblablement de la présence de poignards au sein du mobilier funéraire de ce roi de la XVIII ${ }^{e}$ dynastie. La présente note suggère, après une 
courte description de ces deux objets publiés une première fois en 1904, de les rapprocher d'un type de poignards particulier découvert surtout dans la ville nubienne de Kerma. Une discussion sur les raisons qui ont pu amener un roi de la XVIII ${ }^{e}$ dynastie à emporter des poignards nubiens dans sa tombe est ensuite proposée.

The tomb of Thutmose IV (KV 43), excavated in the early twentieth century by Howard Carter, contained two leather-scabbard fragments, which probably attested to the presence of daggers among the grave goods of the 18th-dynasty king. The present article suggests, after a short description of the two objects published for the first time in 1904, that they are similar to a particular type of dagger discovered in the Nubian city of Kerma in particular. A discussion of the reasons that might have led an 18th-century king to take Nubian daggers to the grave with him follows.

\section{INDEX}

Mots-clés : armement, poignard, fourreau, Thoutmosis IV, XVIIIe dynastie, KV 43, Kerma

Keywords : weaponry, dagger, scabbard, Thutmose IV, 18th dynasty, KV 43, Kerma

\section{AUTEUR \\ RENAUD PIETRI}

Renaud Pietri est actuellement chargé de cours à l'École du Louvre, étudiant en troisième cycle à l'École du Louvre sous la direction de Christophe Barbotin et doctorant en égyptologie à l'université Paul Valéry-Montpellier 3 sous la direction de Luc Gabolde. Il a travaillé sur les poignards, épées et harpés dans l'Égypte ancienne dans le cadre de son Master 2 à l'École du Louvre et mène aujourd'hui des recherches sur la place du char dans la pensée égyptienne dans le cadre de sa thèse de doctorat.

Renaud Pietri is currently a lecturer at the École du Louvre, a postgraduate student under the supervision of Christophe Barbotin at the École du Louvre and studying for a PhD in Egyptology at Université Paul Valéry-Montpellier 3 under the supervision of Luc Gabolde. He has studied daggers, swords and harpēs in ancient Egypt as part of his Master 2 studies at the École du Louvre and is now carrying out research on the place of the chariot in Egyptian thought in the context of his doctoral thesis. 